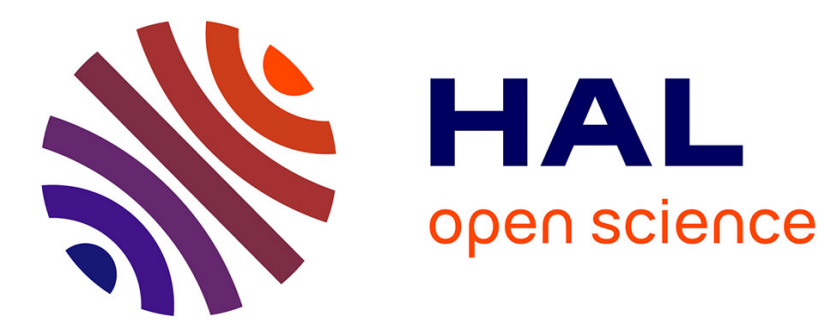

\title{
Conduite de projet, gestion du collectif de travail et unités de soins
}

Pierre-Philippe Dujardin, Thomas Reverdy, Annick Valette, Patrice Francois

\section{To cite this version:}

Pierre-Philippe Dujardin, Thomas Reverdy, Annick Valette, Patrice Francois. Conduite de projet, gestion du collectif de travail et unités de soins. Soins Cadres, 2015, 24 (94), pp.26-28. 10.1016/j.scad.2015.03.005 . hal-01677395

\section{HAL Id: hal-01677395 \\ https://hal.univ-grenoble-alpes.fr/hal-01677395}

Submitted on 17 Jun 2018

HAL is a multi-disciplinary open access archive for the deposit and dissemination of scientific research documents, whether they are published or not. The documents may come from teaching and research institutions in France or abroad, or from public or private research centers.
L'archive ouverte pluridisciplinaire HAL, est destinée au dépôt et à la diffusion de documents scientifiques de niveau recherche, publiés ou non, émanant des établissements d'enseignement et de recherche français ou étrangers, des laboratoires publics ou privés. 


\title{
Conduite de projet, gestion du collectif de travail et unités de soins
}

\author{
Article pour la revue Soins Cadres (2015)
}

Dujardin Pierre-Philippe, Doctorant (Laboratoire TIMC-IMAG), Enseignant-formateur IFCS - CHU Grenoble, CS 10217 - 38043 Grenoble cedex 9

Mail : ppdujardin@chu-grenoble.fr Tél : 04.76.76.51.27

Reverdy Thomas, Maître de conférences en Sociologie (Laboratoire PACTE / CNRS Grenoble-Alpes Université), Grenoble

Valette Annick, Maître de conférences en Sciences de Gestion, (Laboratoire CERAG / CNRS - Grenoble-Alpes Université), Grenoble

François Patrice, PU-PH, Unité d'Evaluation Médicale. (Laboratoire TIMC-IMAG / CNRS Grenoble-Alpes Université), Grenoble

Résumé : Le cadre de santé est considéré comme un pivot organisationnel. Or la littérature n'aborde son rôle que sur un plan opérationnel, dans une relation interindividuelle. Notre étude qualifie la place du cadre dans sa fonction de coordonateur des activités collectives. Deux schémas relationnels sont mis en évidence : la relation triangulée et la relation bipolaire. Des caractéristiques distinguent les deux schémas.

Mots-clefs : Amélioration de la qualité des soins, cadre de santé, conduite de projet, management, activité collective

\section{Introduction}

Depuis près de 20 ans, l'un des buts des réformes hospitalières est de rationaliser l'offre de soins dans un objectif de performance et de qualité. Or la performance est liée au principe même d'organisation et de coordination des activités [1]. Dès les années 1950 Deming [2] constate que $85 \%$ des erreurs sont issues de l'agencement des processus de production et qu'elles dépendent de l'engagement managérial. La coordination des activités collectives est un travail d'encadrement qui consiste à harmoniser les représentations de chacun, répartir les tâches, fixer les règles de fonctionnement, synchroniser les actions avec un objectif de performance [3].

\section{La fonction cadre de santé, un pivot immatériel}

La littérature, professionnelle et scientifique, identifie la place centrale des cadres de santé (CdS) dans le domaine de l'organisation du travail : pilier [4], ossature [5], acteur frontalier [6], pivot [7 - 8], à l'interface de toutes les logiques [6 ; 9]; médiateur institutionnel [10], un travail d'articulation opérationnel incessant [11].

Dans ces différents travaux l'activité de cadre de proximité est abordée sous l'angle opérationnel (corriger des défaillances) et interindividuel (négocier des arrangements). Grosjean et Lacoste [12] montrent que les difficultés organisationnelles ne s'expriment pas collectivement. Bourret [11] confirme cette absence de régulation collective. Elle caractérise la grande variabilité des activités du $\mathrm{CdS}$, dans un travail interstitiel, décousu, invisible et 
interindividuel. Lépine [13] montre la fréquence et la richesse des échanges quotidiens entre le $\mathrm{CdS}$ et chaque membre de l'équipe (présence, disponibilité, soutien) sans référence à une relation structurante collective.

\section{La coordination des activités collectives : une activité professionnelle peu explorée}

Malgré la motivation des $\mathrm{CdS}$ à conduire des projets d'amélioration de la qualité des soins, [11], en adoptant un management participatif, nous ne retrouvons pas dans la littérature de référence à ce travail de coordination des activités dans une dimension collective, qualifié par Strauss [14] et Lacoste [12] d'articulation structurante.

L'objectif de notre contribution est de valoriser le travail des CdS dans la coordination des activités collectives. Pour répondre à cet objectif, nous allons chercher à saisir des problèmes récurrents identifiés par les CdS comme des projets d'amélioration de l'organisation des soins qu'ils souhaitent conduire. Nous considérons que la gestion de projet porte, dans sa définition [15], les éléments d'un travail de coordination collective. Il articule les connaissances distribuées dans un espace de relations individuelles et collectives. Pour explorer la coordination des activités collectives, nous nous appuyons sur un cadre d'analyse proposé par Hatchuel [16], construit à partir de la relation des deux éléments : le sujet (acteur ou groupe d'acteurs) et l'objet (connaissance).

\section{Matériel et méthode}

Dans le cadre d'un Programme Hospitalier de Recherche Infirmière et Paramédicale, s'appuyant sur un dispositif pédagogique, développé à l'Institut de Formation des Cadres de Santé de Grenoble [17], une recherche sur le thème de la conduite de projet est mise en œuvre à partir d'un problème d'organisation défini par un $\mathrm{CdS}$. Un groupe de cinq étudiants cadre, établit une analyse stratégique et propose des actions d'amélioration. Au total, quatorze projets ont fait l'objet d'un suivi sur un an à partir d'entretiens avec les $\mathrm{CdS}$ et des représentants de l'équipe.

\section{Résultats}

Notre recherche identifie deux formes d'utilisation du projet, révélateur de la relation collective entre le CdS et l'équipe (Figure 1) :

- Une relation triangulée (Schéma 1) : Le CdS présente le projet en réunion d'équipe. Les actions proposées par les étudiants sont discutées. L'échange conduit à leur validation, ou à leur refus. Il permet aussi d'identifier d'autres perspectives d'actions, avant leur mise en œuvre. La relation triangulée est retrouvé dans huit projets.

- Une relation bipolaire (Schéma 2) : Le CdS utilise le projet pour mettre en ouvre les actions qu'il a retenues. Bien que l'équipe ait été mise à contribution au moment du travail des étudiants, celle-ci n'est pas informée des conclusions et des suites qui sont données au travail des étudiants. La relation bipolaire concernait six projets. 

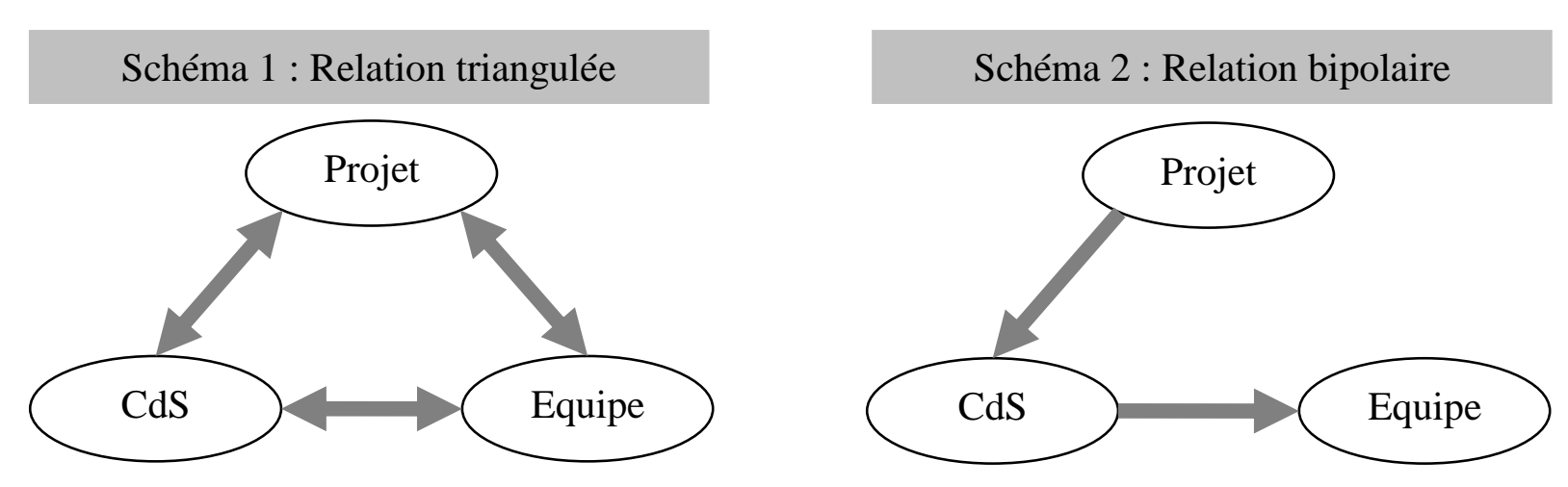

Figure 1 : Modélisation de la relation entre : le projet, le CdS et l'équipe

\section{La relation à l'équipe}

La relation triangulée traduit une symétrie dans la relation avec l'équipe. Ces CdS veulent impliquer leurs équipes dans un processus intellectuel et organisationnel. Ils utilisent les ressources de leurs équipes (compétences professionnelles) en complément des leurs (compétences organisationnelles et transversales ; connaissances individuelles des membres de l'équipe). Cette symétrie de relation s'observe également par le renoncement de certaines actions, pertinentes à leurs yeux, mais refusées par les équipes. Ces $\mathrm{CdS}$ font preuve de patience : "Imposer, ca ne marche pas". Leur stratégie va être d'utiliser les situations problèmes pour argumenter auprès des équipes le bénéfice de l'action. Dans ce schéma relationnel, des réunions d'équipe sont systématiquement retrouvées.

Dans la relation bipolaire, le CdS focalise son attention sur la relation au problème plutôt que la relation à l'équipe. Dans ce schéma, on ne retrouve pas de réunion pour traiter des problèmes d'organisation.

\section{La dimension temporelle}

Dans le schéma triangulé la réflexion collective s'amorce dès la formalisation. Le CdS qualifie son rôle comme "animateur" de l'équipe plus que "décideur". En réalité il laisse s'exprimer la controverse, il régule les échanges en faisant valoir sa position, dans une volonté de consensus autour de la formulation du problème et des perspectives de solutions à mettre en œuvre. L'important nous dira un CdS est de "donner du sens, et faire en sorte de dépasser le simple accord pour aboutir au faire ensemble." Pour le CdS c'est un long et délicat travail. Un long travail car il nécessite des temps collectifs formalisés, combinés à des temps individualisés informels. Les CdS savent que tout ne va pas se dire en réunion. Ils doivent réguler les enjeux interindividuels. Il faut aussi laisser du temps pour une réflexion individuelle. Les CdS disent qu'il faut "laisser mijoter", "maturer" pour permettre cette évolution cognitive. De plus, il n'est pas toujours facile de "désencastrer" les équipes de leurs activités quotidiennes pour les amener sur une dimension moins opérationnelle et plus réflexive.

Dans la relation bipolaire, la dimension temporelle concerne uniquement la réflexion personnelle du CdS. Une fois qu'il a cerné le problème et sa capacité d'action pour le résoudre, il met en œuvre! L'équipe est mise à contribution pour appliquer la décision dans l'instant. S'il n'y a pas d'exécution, le cadre "rabâche". De son coté, l'équipe, sans être dans la résistance, ne 
comprend pas toujours ce que le CdS demande. De cette incompréhension, nait une tension entre le CdS et l'équipe. Cette dernière souhaiterait d'ailleurs plus d'échanges collectifs, et pointe les limites d'un management informel.

\section{Discussion}

La gestion de projet conduite par les CdS est un objet de recherche qui permet de qualifier leur contribution à l'organisation structurante.

Nos résultats montrent que la réunion est l'espace d'échange collectif utilisé par les CdS pour développer une articulation structurante des savoirs distribués. Elle permet la mise en application des principes de l'ingénierie concourante, c'est à dire la complémentarité des ressources cognitives. Ces $\mathrm{CdS}$ valorisent les expertises professionnelles, placent leurs compétences dans une dimension managériale et non soignante. Il s'agit d'un travail d'ajustement entre le $\mathrm{CdS}$ et l'équipe qui consiste à un "frayage pré-décisionnel". Pour d'autres $\mathrm{CdS}$, l'absence de réunion est une volonté délibérée. Ils préfèrent adopter un management plus informel car la réunion est une prise de risque : le risque de se retrouver seul face à l'équipe, sans réponse aux incohérences institutionnelles [11] et sans soutien hiérarchique [13]. La réunion conduirait à mettre au jour une forme d'impuissance managériale à traiter ces problèmes d'organisation $[13 ; 18]$.

Les schémas relationnels, mis en évidence dans notre étude, s'approchent de la conception de l'action managériale qualifiée par Habermas [19] : d'un "agir orienté vers l'intercompréhension" pour la relation triangulée ; d'un "agir orienté vers le succès" pour la relation bipolaire.

Cette dualité, entre résoudre ou comprendre, s'exprime également dans la rapidité d'exécution. Certains CdS sont dans une mise en œuvre très rapide des actions et utilisent le travail des étudiants comme une prescription. D'autres se donnent du temps, et mettent de la distance avec le rapport fournit par les étudiants. Le point de vue extérieur est une base pour susciter la réflexion de l'équipe et du CdS.

On touche ici à la capacité des $\mathrm{CdS}$ à résister à la pression temporelle : pression d'une institution sur les CdS, qu'ils répercutent sur les équipes [11]. Or, minimiser la dimension temporelle c'est prendre le risque d'essuyer un échec [20], en mettant l'équipe en opposition. Ne pas investir du temps de coordination, c'est courir le risque de ne pouvoir faire face à l'accélération des rythmes de prise en charge [18] et au final, accroitre davantage les dysfonctionnements. Plusieurs $\mathrm{CdS}$ se disent résignés de ne pouvoir traiter les questions organisationnelles dans une dimension collective : "Il n'y a plus de temps pour se poser". Pour eux, il n'est pas concevable d'instaurer des temps de travail improductifs, dans un contexte où il est difficile de répondre aux activités productives de soins. Les CdS font des choix. Ils hiérarchisent les priorités avec au premier rang les activités de soins. Ils évaluent le coût d'une réunion en heures de travail pour chaque membre de l'équipe face au cumul des heures supplémentaires qu'ils n'arrivent pas à absorber. Au final, dans ce schéma, c'est toujours la coordination collective qui en pâtit. 


\section{Conclusion}

Bien que nécessaire, l'efficacité ne s'obtient pas par le travail macroscopique au niveau institutionnel. L'efficacité dépend du travail de coordination des opérateurs de production au niveau microscopique [21], et le rapport entre l'articulation opérationnelle et l'articulation structurante dans une recherche de stabilité et de gain de temps [12]. C'est au cadre de proximité que revient ce travail de coordination structurante, "figure d'entrepreneur organisationnel" [21]. Pour ce faire il est nécessaire de développer des espaces de coordination collective qui font cruellement défaut dans les organisations hospitalières, pour que puisse s'exprimer la controverse [22].

Les problèmes rencontrés dans les organisations sont des problèmes d'ajustement complexes qui doivent passer par des espaces de coordination collective structurés [21]. Le fait qu'il y ait controverse entre les acteurs ne doit pas être un motif de renoncement des $\mathrm{CdS}$ à l'organisation de ces temps d'échange, mais au contraire être considéré comme une communication opérante suscitant un débat de fond dans la perspective d'un consensus au bénéfice d'une organisation performante.

\section{Bibliographie}

[1] Lorino $\mathrm{Ph}$, Tarondeau JC. De la stratégie aux processus stratégiques. Revue française de gestion $2006 ; 160: 307-328$

[2] Deming W.E. Hors de la crise. Paris : Economica 1991

[3] Barthe B, Queinnec Y. Terminologie et perspectives d'analyse du travail collectif en ergonomie. L'année psychologique 1999 ; 99.4. : 663-686

[4] Dumas M, Ruiller C. Quelles compétences pour le cadre de santé de demain ? Entre prescriptions multi-objectifs et réalité du terrain : un métier à "panser". 22ème Congrès de l'AGRH 2011

[5] Saint-Marc T, Fischler M, Tillon-Faure O. Bilan à deux ans de la mise en place de pôles d'activité. Gestions Hospitalières (3) 2010 ; 494 : 137-151

[6] Rivière A. Tensions de rôle et stratégies d'ajustement : une étude auprès de cadres de santé à l'hôpital. Journal de Gestion et d'Économie Médicales 2013 ; 31.2 : 142-162

[7] De Singly C. Rapport de la Mission Cadres Hospitaliers. Ministère de la Santé et des Sports 2009

[8] Hees M. Le management à l'hôpital : Science, art ou magie ? Coll. Perspective soignante, Paris : Seli Arslan 2010

[9] Coulon R. Le cadre de santé entre logiques d'utilité et logiques de soins hospitaliers, 2ème journée transdisciplinaire de recherche en Management Hospitalier 2010

[10] Svandra P. Entre référent et médiateur : la légitimité du cadre soignant. In : L'essentiel du management, Rosenblatt F. Coll. Objectif soins, Rueil-Malmaison : Lamarre 2006 : 55-56

[11] Bourret P. Les cadres de santé à l'hôpital. Un travail de lien invisible. Paris : Seli Arslan 2006 
[12] Grosjean M, Lacoste $M$. Communication et intelligence collective. Le travail à 1’hôpital. Coll. Le Travail Humain, Paris : PUF 1999

[13] Lépine V. La reconnaissance au travail par la construction d'une relation agissante : la communication des cadres de santé. Revue Communication \& Organisation (12) 2009 ; 36 : 97-108.

[14] Strauss A, La trame de la négociation. Paris : L'Harmattan 1992

[15] ANACT. L'organisation et le management "par projet". ANACT 2006

[16] Hatchuel A. Pour une épistémologie de l'action. L'expérience des sciences de gestion. In : Entre connaissance et organisation : l'activité collective. Teulier R, Lorino P. Coll. Recherches, Paris : La Découverte 2005 : 72-92

[17] Dujardin PP, Raynal V, Molinaro A, Vissac C, Capron D, Cholez C. Conduite de projet pour préparer l'avenir. Soins Cadres (8) 2011 ; 79: 38-40.

[18] Detchessahar M. Construire la qualité de vie au travail à l'hôpital, soigner le management. Soins Cadres (2) 2013 ; 85 : 28-32

[19] Habermas J. Théorie de l'agir communicationnel. Tome 1. Rationalité de l'agir et rationalisation de la société. Paris : Fayard 1987

[20] Husser J. Le pilotage des équipes hospitalières par le management quotidien d'articulation. Vie \& sciences de l'entreprise $2011 ; 189$ : 23-45

[21] Moisdon JC. Gouvernance clinique et organisation des processus de soins : un chaînon manquant ? Pratiques et Organisation des Soins (7-9) 2008 ; 39.3 :175-181

[22] Herreros G, Milly B. Les voies de la qualité à l'hôpital : entre procédures et coopération. (4) 2006

Tableau 1 : synthèse des caractéristiques des CdS en fonction du schéma relationnel

\begin{tabular}{lcc}
\hline Caractéristiques & Relation triangulée & Relation bipolaire \\
\hline Principe relationnel du CdS & A équipe (symétrie) & Au problème \\
Réflexion & Collective (équipe et CdS) & Personnelle du CdS \\
Espace relationnel & Réunion organisationnelle & Management informel \\
Sens de l'agir managérial & Orienté vers l'intercompréhension & Orienté vers le succès \\
Utilisation du travail étudiant & Réflexion & Prescription \\
Temporalité & Long terme & Court terme \\
\hline
\end{tabular}

